\title{
Editorial
}

DOI: https://doi.org/10.14483/22487085.13714

\section{Community-Based Approaches to Foreign Language Education}

Learning how to recognise and make student and community assets the subject of curriculum is at the core of teachers' designs and enactments of critical and inclusive pedagogies. However, this era of globalisation and standardisation, where education is increasingly seen as a commodity that underscores economic competitiveness, has made space for local knowledge production, hard to find. Knowing how to incorporate community problems in school-based student-led inquiries, whilst meeting authorised learning outcomes, is also challenging. At the same time there are particular pressures on language teachers where states extol the benefits of English, or another foreign language literacy for global competitiveness. Yet, educational researchers and teacher educators know the potential power of working with students' assets and motivations to enhance language and literacy learning in classrooms. Community based approaches to language education in various places in Latin America are explored in this issue with contributions from teacher-researchers, collaborative teams, teacher educators and university-based researchers.

The writers in this issue draw variously from rich research traditions in sociolinguistics, educational ethnography (Moll, Amanti, Neff $\varepsilon$ Gonzalez, 1992), teacher-research (CochranSmith $\varepsilon$ Lytle, 2009), collaborative inquiry and school-based experiments to portray a rich picture of innovative research in language and literacy education which promotes the second language learner as competent and agentive. From Bogotá, to Villanueva, and Mexico, authors document the work of teachers, student teachers and students engaging with local knowledge and problems whilst learning an additional academic language. Read together, these articles demonstrate the resourcefulness of these researchers in working collaboratively with teachers and communities. The issue includes designs which foreground intergenerational learning, testing out of language theory in situ, exploring and documenting local identities, knowledges, practices and histories and developing critical language educators.

Nohora Inés Porras, Lenys Smith Díaz and Marlen María Nieves report on a fascinating approach to building language teaching expertise by partnering student teachers with expertise in English language teaching with experienced teachers with limited experience and facility with English in Bucaramanga, Colombia. Both preservice and in-service teachers benefit from the reciprocal mentoring which emerges. The in-service teachers access a range of strategies and techniques for engaging their classes with English language learning from the student teachers, who themselves gain valuable insights in classroom management and building relationships with elementary school learners. Their approach combines reverse mentoring and peer coaching, thereby positioning teachers, at different stages of career, as contributors to each other's pedagogical repertoires, whilst collaborating to enhance children's opportunities for English language engagement. In previous studies I have also found cross-generational reciprocal mentoring a very powerful way of countering the deficit discourses that sometimes circulate in marginalised and poor communities where English language and literacy cannot be taken-for-granted (Comber $\mathcal{E}$ Kamler, 2004). This is an untapped resource in many places as different generations go under-recognised for what they might know and how they might contribute to each other and towards solving problems.

The potential of young learners is highlighted in the article by Kristen Lindahl and Peter Sayer. Their experimental study in 4 schools in Mexico (urban, suburban and rural) sought to test 
'the more and earlier approach' which is informed by linguistic interdependence theorising (Cummins, 1979), which suggests that first language literacy may benefit from early learning of another language. This study confirmed the linguistic interdependence hypothesis with significant implications for policy for language education in Mexico, namely support for a 'program designed to give simultaneous exposure to English and Spanish from kindergarten'. However, the authors do point that further research is needed on the kinds of language pedagogies which foster linguistic interdependence in different contexts. Further, they question the assumption that more English literacy necessarily leads to socio-economic mobility for students from low socio-economic families. These are important questions internationally. On the one hand we are reminded that young children have great capacities for language learning; on the other hand, we know that language and literacy repertoires, in and of themselves, are insufficient to rectify serious material inequalities.

Alba Milena Flórez documents an action research study with her class of young adults (15-18 years of age) from Villanueva who were learning English as a Foreign Language. As a teacher-researcher, Flórez negotiated a community-based program, where her students became researchers and chroniclers of elders' accounts of the development of Villanueva as an independent municipality. The complexity of the community studies approach saw Flórez's students co-designing an interview protocol in English, translating it into Spanish for their elderly informants, organising ethics consent and ultimately working together to produce a chronicle about the town. This ambitious project not only engaged all the participants it also produced clear gains in their use of English. As well as the positive impact on the students and their interlocutors, the project led to a new level of critical reflexivity on the part of the researcher. Flórez includes a truly fascinating table concerning her own pedagogical practices before and after the intervention. This will be of great interest and value to other teacher-researchers, as we see her move from a teacher-centred approach to language teaching to truly negotiating the curriculum (Boomer, 1999). As Flórez recognizes, this shift meant that her students had quite different opportunities for rich and meaningful language learning. Ultimately, the community-based approach allowed students to develop a sense of local identity, acquire transferrable study techniques, and improve their own academic literacies in English.

Sandra Patricia Lastra, Norma Constanza Durán and Deyssi Acosta also worked with a community based pedagogical approach in their collaborative research with students in an English undergraduate program at the University of Tolima, Ibagué, Colombia. The researchers worked with their students to develop understandings about community funds of knowledge, local knowledge and the idea of the community teacher. A key activity was for students to appreciate the notion of 'community assets'. The team began by having students explore and map their own university community. This conscious-raising task made students realise who and what they took for granted as part of their everyday lives. Five students went on to design a small-scale community-based project to conduct with the community during their practicum. Student teachers began to realise their own responsibility in recognising and understanding the realities of the communities in which their practicum was to be situated. They began to recognise their potential roles as advocates and change-makers in achieving student rights. While the student-teachers' research projects were modest due to time constraints, they had begun to take an inquiry stance (Cochran-Smith $\&$ Lytle, 2009) and to foreground equity issues in their planning, and to start from the students' dynamic and changing lifeworlds in imagining their curriculum. 
Juliana Llanes undertook a case study approach to developing cultural competence with Asian students attending the Foreign Language Department at the University in Bogotá who were learning Spanish as a Foreign Language. The students initially volunteered to work with local people in libraries and homes for the elderly. As part of their program they also expressed interest in learning about farming and regional communities. One student made strong connections with a rural cook and together they explored their preferences for food and its preparation. Another also had conversations about food and coffee farming. Students found that in conversing with people about matters of everyday life and local culture and traditions, they were motivated to find the words to express their meanings. Together with local workers they co-constructed cultural knowledge.

In an action research study with fifth grade students, in a public school in Bogotá, Ana Janeth Gómez discovered that student inquiries into 'situations of conflict' could become 'opportunities in life that could be faced for living better'. She also found that her students simultaneously engaged in translanguaging to express their ideas in Spanish and English language about getting on together in the school. This inspiring teacher research reminds us that language is best learned in the process of getting other things done in the world. What the world would appear to need more than anything else is people who can learn to coexist and collaborate. In this study it becomes clear that school children are already citizens working, learning and playing together; they are not simply rehearsing for a possible future. In this project we see how school inquiry can help children and teachers learn better by working together - the assets of the group are more than the sum of its parts. This requires a wise and responsive teacher, like Gómez, who is equally open to learning.

In a similar vein, Yuly Nieto reviews the literature on the ways local literacies can be incorporated into language teacher education. Nieto argues that the community itself can be seen as a text to be explored by teachers and students. Understanding the community's assets is crucial to designing community-based pedagogies. This approach is particularly suitable to student teachers as they assemble the resources they need for responding to students and towards developing a teacher standpoint and identity. Such an approach also lends itself to a transdisciplinary problem-based curriculum which can attend to academic knowledge and local knowledges in situ as relevant (see also Sharkey, Clavijo and Ramirez, 2016). Accessing an alternative justice-driven discourse is increasingly important in the context of government promoted standardized discourses of teacher excellence, as María Eugenia Plata Santos notes in her article. Interrogating the discursive construction of the 'quality teacher' is urgent for critical educators world-wide (Scholes, Lampert, Burnett, Comber $\&$ Hoff, 2017). The fields of educational and social science are increasingly globalised with the multinational publication apparatuses of the North dominating citation indexes. In these circumstances it is even more obvious that who gets to produce knowledge faces a major challenge if he/she belongs to states who are not at the centre, but on the periphery. As Miguel Farías uncovers, the work of scholars in the humanities, such as applied linguistics in education for example, is imperative to sustaining critical scholarship at a time when global measures is what counts as scholarship threaten to overwhelm counter-hegemonic discourses.

This collection of articles gives us reason to hope. As a critical literacy educator working with teacher-researchers in poor and regional Australia, I see huge potential for transnational dialogues between researchers and front-line practitioners in language education. Getting this important work out is more urgent than ever! I thank the contributors and the editors of the Colombian Applied Linguistics Journal for sharing this powerful work with me. I will send it on to friends and colleagues educating for social justice. 


\section{References}

Boomer, G. (1999). Changing curriculum. In B. Green (Ed.) Designs on learning: Essays on curriculum and teaching by Garth Boomer. Deakin West, ACT: Australian Curriculum Studies Association.

Cochran-Smith, M. E Lytle, S. (2009). Inquiry as Stance: Practitioner Research for the Next Generation. New York, NY: Teachers College Press.

Comber, B. E Kamler, B. (2004). Getting out of deficit: Pedagogies of reconnection. Teaching Education, 15(3), 293-310. https://doi.org/10.1080/1047621042000257225

Cummins, J. (1979). Linguistic interdependence and the educational development of bilingual children. Review of Educational Research, 49(2), 222-251. https://doi.org/10.3102/00346543049002222

Moll, L., Amanti, C., Neff, D., \& Gonzalez, N. (1992). Funds of knowledge for teaching: Using a qualitative approach to connect homes and classrooms. Qualitative Issues in Educational Research Theory into Practice, 31(2), 132-141. https://doi.org/10.1080/00405849209543534.

Sharkey, J., Clavijo, A., E Ramirez, M. (2016). Developing a deeper understanding of communitybased pedagogies with teachers: Learning with and from teachers in Colombia. Journal of Teacher Education 67(3), 1-14. https://doi.org/10.1177/0022487116654005.

Scholes, L. Lampert, J. Burnett, B. Comber, B \& Hoff, L. (2017). The Politics of Quality Teacher Discourses and Implications for Teachers in High Poverty Schools. Australian Journal of Teacher Education. 42(4), 19-43. https://doi.org/10.14221/ajte.2017v42n4.3.

\section{Barbara Comber PhD UNiversity of South Australia Guest Editor}




\section{Editorial}

DOI: https://doi.org/10.14483/22487085.13714

\section{Enfoques Comunitarios para la Educación en Lenguas Extranjeras}

Aprender a reconocer y hacer de los recursos de los estudiantes y de la comunidad el tema del currículo constituye el núcleo de los diseños y promulgaciones de los maestros de pedagogías críticas e inclusivas. Sin embargo, esta era de globalización y estandarización, en la que la educación se considera cada vez más como una mercancía que subraya la competitividad económica, ha hecho que sea difícil encontrar espacio para la producción de conocimientos locales. Saber cómo incorporar los problemas de la comunidad en las investigaciones dirigidas por los estudiantes en las escuelas, a la vez que se alcanzan los resultados de aprendizaje requeridos, también es un desafío. Al mismo tiempo, existen presiones particulares sobre los profesores de lenguas en los que el estado resalta los beneficios de saber inglés u otro idioma extranjero para la competitividad global. Sin embargo, los investigadores en educación y los formadores de docentes conocen el potencial de trabajar con estudiantes, sus recursos y sus motivaciones para mejorar el aprendizaje del lenguaje y las literacidades en las aulas. En este número se exploran los enfoques comunitarios en la enseñanza de lenguas en varios lugares de América Latina, con contribuciones de docentes investigadores, equipos de investigadores, formadores de docentes e investigadores universitarios.

Los autores de este número se inspiran en las tradiciones de investigación en sociolingüística, etnografía educativa (Moll, Amanti, Neff y González, 1992), investigación docente (CochranSmith y Lytle, 2009), investigación colaborativa y experimentos en las escuelas para presentar un panorama rico de investigación innovadora en el campo del lenguaje y las literacidades que promueve al estudiante de una segunda lengua como competente y agente activo de su propio aprendizaje. En Colombia, desde Bogotá hasta Villanueva, Santander y México, los autores documentan el trabajo de docentes, estudiantes en práctica pedagógica y estudiantes que se involucran con el conocimiento y los problemas locales mientras aprenden una lengua extranjera. Vistos en conjunto, estos artículos demuestran la capacidad de los investigadores para trabajar en colaboración con los maestros y las comunidades. Este número incluye diseños que ponen en primer plano el aprendizaje intergeneracional, comprobando teorías del lenguaje en la escuela, explorando y evidenciando identidades locales, conocimientos, prácticas e historias locales, y desarrollando educadores críticos.

Nohora Inés Porras, Lenys Smith Díaz y Marlen María Nieves escriben sobre un enfoque fascinante para construir la experiencia en la enseñanza de lenguas al asociar a estudiantes de práctica docente con experiencia en la enseñanza del inglés con profesores de primaria con poca experiencia en inglés en Bucaramanga, Colombia. Tanto los estudiantes de práctica docente como los docentes en ejercicio se benefician de la tutoría recíproca que surge. Los docentes en ejercicio tienen acceso a una variedad de estrategias y técnicas para involucrar a sus estudiantes en el aprendizaje de inglés a través de la experiencia que les ofrecen los practicantes, quienes a su vez adquieren valiosos conocimientos en manejo de aula y sobre formas de construir relaciones con los estudiantes de primaria. Su enfoque combina la tutoría inversa y la tutoría de pares, posicionando así a los docentes, en diferentes etapas de su carrera profesional, como apoyo al desarrollo de los repertorios pedagógicos de ambos, a la vez que colaboran para mejorar las oportunidades de aprendizaje de inglés de los niños. En estudios previos que he realizado, también he encontrado que la tutoría recíproca intergeneracional es una manera muy poderosa de contrarrestar los discursos de déficit que a veces circulan en comunidades marginadas y pobres donde el aprendizaje del inglés y el 
desarrollo de la literacidad no pueden darse por hecho (Comber $E$ Kamler, 2004). Este es un recurso sin explotar en muchos lugares, ya que los saberes de las diferentes generaciones no son reconocidos ni valorados por lo que cada generación puede saber y por la forma en que ésta puede contribuir a otras generaciones a la solución de problemas educativos.

El potencial de los jóvenes estudiantes se destaca en el artículo de Kristen Lindahl y Peter Sayer. Su estudio experimental en 4 escuelas en México (urbanas, suburbanas y rurales) buscó probar "el enfoque de más inglés y más temprano" que se basa en la teoría de la interdependencia lingüística (Cummins, 1979), lo que sugiere que si el desarrollo de literacidad en la primera lengua puede beneficiar el aprendizaje temprano de otra lengua. Este estudio confirmó la hipótesis de la interdependencia lingüística con implicaciones significativas para la política de educación en México, a saber, el apoyo a un "programa diseñado para dar exposición simultánea al inglés y al español desde el preescolar". Sin embargo, los autores señalan que se necesita más investigación sobre los tipos de pedagogías del lenguaje que fomentan la interdependencia lingüística en diferentes contextos. Además, cuestionan la suposición de que una mayor literacidad en inglés conduce necesariamente a la movilidad socioeconómica de los estudiantes de familias de bajos ingresos. Estas son cuestiones importantes a nivel internacional. Por un lado, se nos recuerda que los niños pequeños tienen grandes capacidades para el aprendizaje de lenguas; por otro lado, sabemos que los repertorios de lenguaje y literacidad, por sí solos, son insuficientes para transformar las graves desigualdades económicas.

Alba Milena Flórez documenta un estudio de investigación acción con su clase de adolecentes (15-18 años de edad) de Villanueva quienes están aprendiendo inglés como lengua extranjera. Como profesora investigadora, Flórez propuso e implementó un programa basado en la comunidad, donde sus estudiantes se convirtieron en investigadores y cronistas de los relatos de los ancianos sobre el desarrollo de Villanueva como municipio independiente. La complejidad del enfoque de los estudios comunitarios hizo que los estudiantes de Flórez co-diseñaran un protocolo de entrevista en inglés, lo tradujeran al español para los adultos mayores como sus informantes, y firmaran un consentimiento informado para cumplir con el código de ética y finalmente trabajaran juntos para producir una crónica sobre el municipio. Este ambicioso proyecto no sólo involucró a todos los participantes, sino que también produjo claros beneficios en el uso del inglés. Además del impacto positivo en los estudiantes y sus interlocutores, el proyecto llevó a un nuevo nivel de reflexividad crítica por parte del investigador. Flórez incluye una plataforma realmente fascinante sobre sus propias prácticas pedagógicas antes y después de la intervención. Esto será de gran interés y valor para otros profesores-investigadores, ya que su práctica pedagógica se transforma de un enfoque centrado en el docente a la construcción de un currículo con estudiantes para la enseñanza del inglés (Boomer, 1999). Como reconoce Flórez, este cambio significó que sus estudiantes tuvieron oportunidades muy diferentes para un aprendizaje de lenguas rico y significativo. En última instancia, el enfoque basado en la comunidad permitió a los estudiantes desarrollar un sentido de identidad local, adquirir técnicas de estudio transferibles y mejorar sus propias literacidades académicas en inglés.

Sandra Patricia Lastra, Norma Constanza Durán y Deyssi Acosta también trabajaron con un enfoque pedagógico basado en la comunidad en su investigación colaborativa con estudiantes en un programa de licenciatura en inglés en la Universidad del Tolima, Ibagué, Colombia. Los investigadores trabajaron con sus estudiantes para desarrollar comprensión sobre los fondos de conocimiento de la comunidad, conocimiento local y la idea del maestro comunitario. Una actividad clave fue que los estudiantes apreciaran la noción de "recursos de la comunidad". 
El equipo comenzó haciendo que los estudiantes exploraran y trazaran un mapa de su propia comunidad universitaria. Esta tarea de concientización hizo que los estudiantes se dieran cuenta de lo que daban por hecho como parte de su vida cotidiana. Cinco estudiantes diseñaron un proyecto a pequeña escala para llevar a cabo con la comunidad durante su práctica docente. Los practicantes empezaron a darse cuenta de su propia responsabilidad en el reconocimiento y la comprensión de las realidades de las comunidades en las que se iba a situar su práctica docente. Comenzaron a reconocer su rol potencial como defensores y hacedores de cambios en el logro de los derechos de los estudiantes. Mientras que los proyectos de investigación de los estudiantes y profesores eran modestos debido a las limitaciones de tiempo, ellos habían comenzado a tomar una postura de investigación (Cochran-Smith $\varepsilon$ Lytle, 2009) y a poner en primer plano las cuestiones de equidad en su planeación y a partir de la dinámica de los estudiantes y sus realidades cambiantes para imaginar el currículo.

Juliana Llanes emprendió un estudio de caso para desarrollar la competencia cultural con estudiantes asiáticos que asistían al Departamento de Lenguas Extranjeras de una Universidad de Bogotá y que estaban aprendiendo español como lengua extranjera. Inicialmente, los estudiantes se ofrecieron como voluntarios para trabajar con la población local en bibliotecas y hogares para ancianos. Como parte de su programa, también expresaron interés en aprender sobre la agricultura y las comunidades regionales. Un estudiante hizo fuertes conexiones con un cocinero rural y juntos exploraron sus preferencias por la comida y su preparación. Otro también tuvo conversaciones sobre el cultivo de alimentos y café. Los estudiantes descubrieron que al conversar con la gente sobre temas de la vida cotidiana y la cultura y tradiciones locales, se sentían motivados para encontrar las palabras que expresaran sus significados. Junto con los trabajadores locales, ellos co-construyeron el conocimiento cultural.

En un estudio de investigación acción con estudiantes de quinto grado, en una escuela pública de Bogotá, Ana Janeth Gómez descubrió que las investigaciones de los estudiantes sobre "situaciones de conflicto" podrían convertirse en "oportunidades en la vida que se podrían enfrentar para vivir mejor". También descubrió que sus estudiantes se dedicaban simultáneamente a traslanguaging para expresar sus ideas en español y en inglés sobre cómo trabajar juntos en la escuela. Esta investigación de aula inspiradora realizada por la maestra nos recuerda que el lenguaje se aprende mejor utilizándolo en el hacer cosas que tienen relación con nuestra vida. Lo que el mundo parece necesitar más que nada son personas que puedan aprender a coexistir y colaborar. En este estudio queda claro que los estudiantes de quinto grado ya se piensan así mismos como ciudadanos cooperando, aprendiendo y jugando juntos; no están simplemente ensayando para un futuro posible. En este proyecto vemos cómo la investigación escolar puede ayudar a los niños y a los docentes a aprender mejor compartiendo juntos: los recursos del grupo son más que la suma de sus partes. Esto requiere un maestro sabio y receptivo, como Gómez, que esté igualmente abierto al aprendizaje.

Igualmente, Yuly Nieto revisa la literatura sobre las formas en que las literacidades locales pueden ser incorporadas a la formación de los profesores de lenguas. Nieto argumenta que la comunidad misma puede ser vista como un texto para ser explorado por docentes y estudiantes. Comprender los recursos de la comunidad es crucial para diseñar pedagogías basadas en la comunidad. Este enfoque es particularmente adecuado para los estudiantes en práctica docente, para que ellos utilicen los recursos que necesitan para responder a los estudiantes y para desarrollar un punto de vista e identidad docente. Tal enfoque 
también se presta a un currículo transdisciplinario basado en problemas que puede atender al conocimiento académico y al conocimiento local en la escuela según sea relevante (ver también Sharkey, Clavijo y Ramírez, 2016). El acceder a un discurso alternativo basado en la justicia es cada vez más importante en el contexto de los discursos estandarizados de excelencia docente promovidos por el gobierno, como señala María Eugenia Plata Santos en su artículo. Interrogar la construcción discursiva del "maestro de calidad" es urgente para los educadores críticos de todo el mundo (Scholes, Lampert, Burnett, Comber $\mathcal{E}$ Hoff, 2017). En los campos de la educación y las ciencias sociales que son cada vez más globalizados, es un logro producir conocimiento a pesar del desafío que representa no ser parte del sistema económico de las publicaciones financiadas del Norte. Como lo descubre Miguel Farías, el trabajo de los expertos en las humanidades, como la lingüística aplicada a la educación, es imperativo mantener una escolaridad crítica en tiempos en que la medición de la academia amenaza los discursos contra-hegemónicos.

Esta colección de artículos nos da motivos de esperanza. Como educadora de literacidad crítica trabajando con profesores-investigadores en regiones de Australia pobres, veo un enorme potencial para el diálogo transnacional entre investigadores y profesionales de primera línea en la educación lingüística. iHacer visible este importante trabajo es hoy más urgente que nunca! Agradezco a los autores y a la editora de la Revista Colombian Applied Linguistics por compartir conmigo este valioso trabajo. Se lo enviaré a amigos y colegas que educan para la justicia social.

\section{References}

Boomer, G. (1999). Changing curriculum. In B. Green (Ed.) Designs on learning: Essays on curriculum and teaching by Garth Boomer. Deakin West, ACT: Australian Curriculum Studies Association.

Cochran-Smith, M. E Lytle, S. (2009). Inquiry as Stance: Practitioner Research for the Next Generation. New York, NY: Teachers College Press.

Comber, B. E Kamler, B. (2004). Getting out of deficit: Pedagogies of reconnection. Teaching Education, 15(3), 293-310. https://doi.org/10.1080/1047621042000257225

Cummins, J. (1979). Linguistic interdependence and the educational development of bilingual children. Review of Educational Research, 49(2), 222-251. https://doi.org/10.3102/00346543049002222

Moll, L., Amanti, C., Neff, D., E Gonzalez, N. (1992). Funds of knowledge for teaching: Using a qualitative approach to connect homes and classrooms. Qualitative Issues in Educational Research Theory into Practice, 31(2), 132-141. https://doi.org/10.1080/00405849209543534.

Sharkey, J., Clavijo, A., \& Ramirez, M. (2016). Developing a deeper understanding of communitybased pedagogies with teachers: Learning with and from teachers in Colombia. Journal of Teacher Education 67(3), 1-14. https://doi.org/10.1177/0022487116654005.

Scholes, L. Lampert, J. Burnett, B. Comber, B \& Hoff, L. (2017). The Politics of Quality Teacher Discourses and Implications for Teachers in High Poverty Schools. Australian Journal of Teacher Education. 42(4), 19-43. https://doi.org/10.14221/ajte.2017v42n4.3.

\section{Barbara Comber PhD \\ University of South Australia \\ EDITORA INVITADA}

